كزئش مهمترين صفات موثر بر عملكرد زنوتيتهاى كنجد (Sesumum indicum L.) در دو شرايط بدون تنش و تنثرد

\author{
افسون عسكرى '، محمد ضابط'، محمد قادر قادرى '، عليرضا صمدزاده 'ّ على شوروزدى \\ 1- دانشجوى كارشناسى ارشد، دانشكاه بيرجند، (نويسنده مسوول: askari_2505@yahoo.com

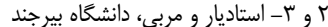

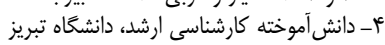

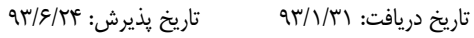

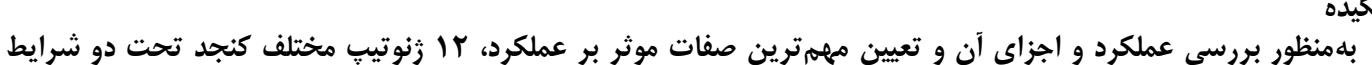

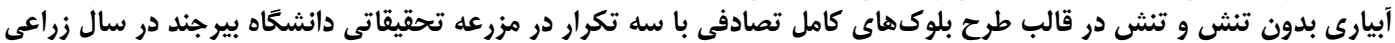

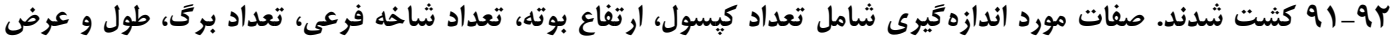

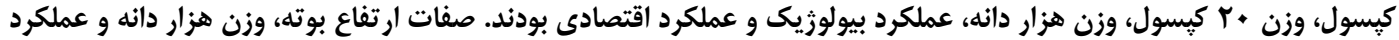

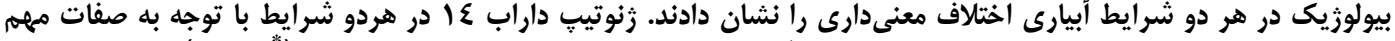

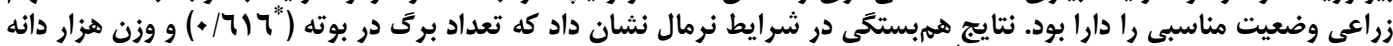

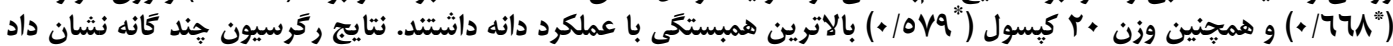

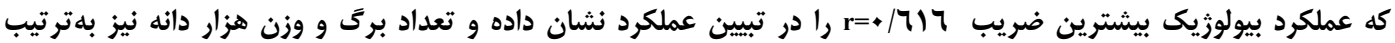

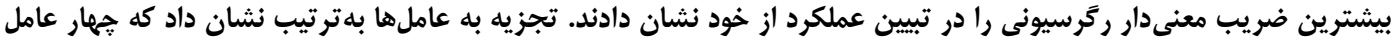

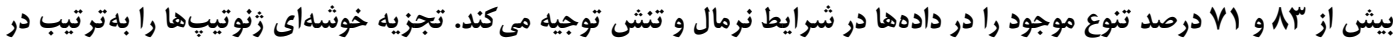

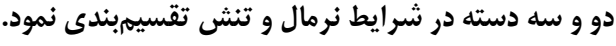

وازههاى كليدى: تجزيه به عاملها، تجزيه رَّرسيون، تجزيه خوشهاى، تجزيه همبستكى، كنجد

و تعرق و محدوديت منـابع آبسى در طـول دوره رشــ كَّاهـان

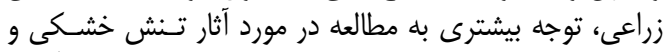

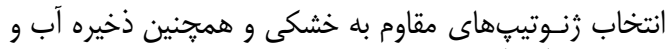

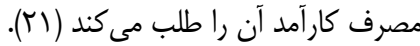

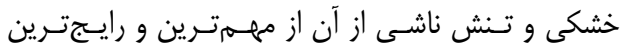

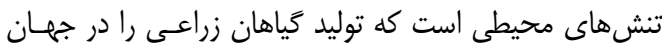

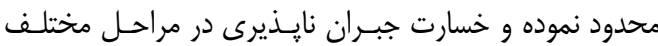

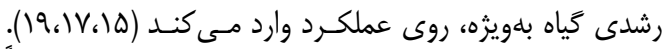

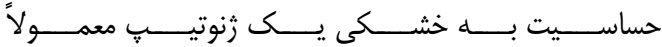

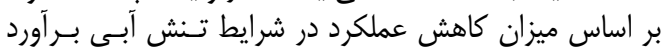

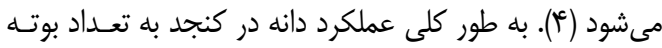

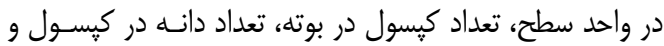

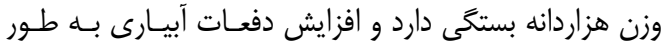

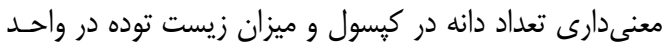

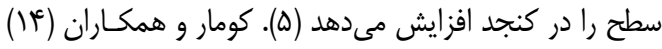

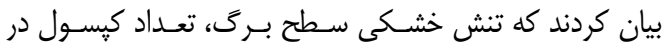

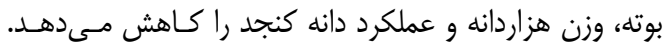

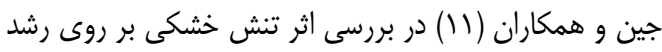

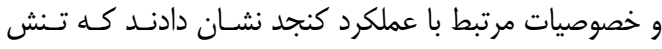

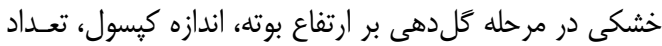

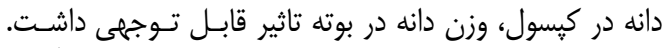

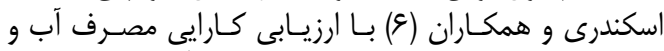

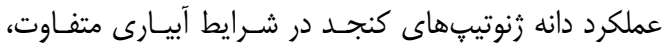

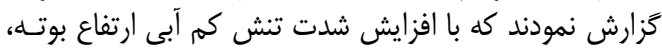

مقدمه - مقان

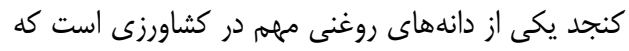

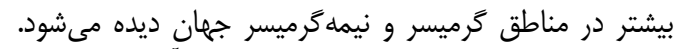

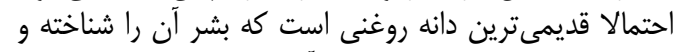

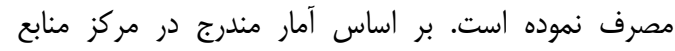

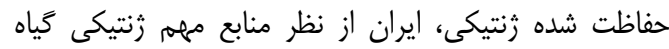

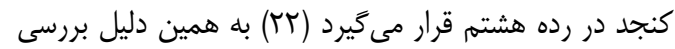

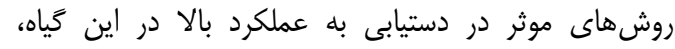

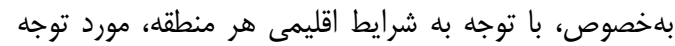

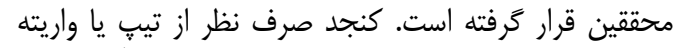

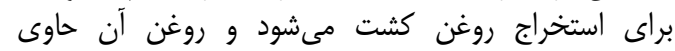

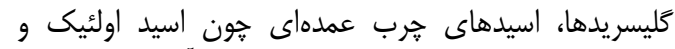

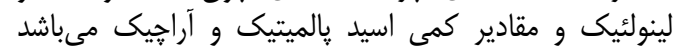

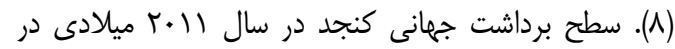

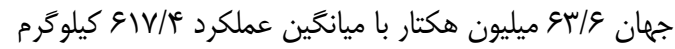

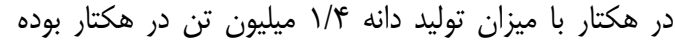

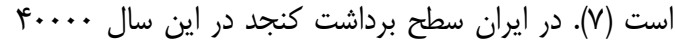

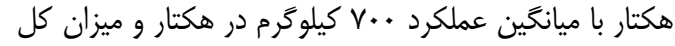

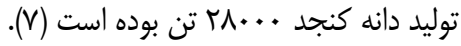

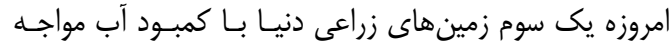

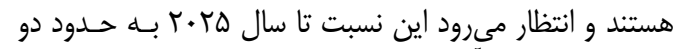

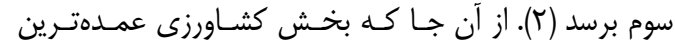

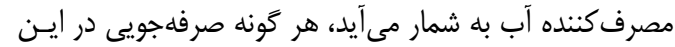
بخش صرفهجويى در منابع آب تلقى مى شود. زئ زياد بودن تبخير 
بافت خاك شنى- لومى بود- اجرا شد. زنوتيڤهاى مورد

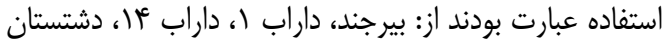

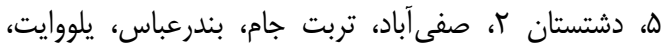

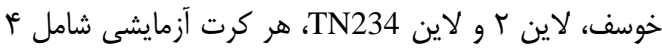

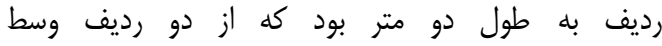

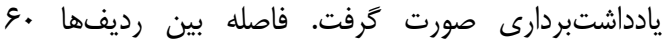

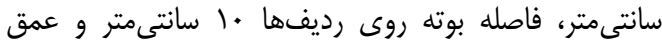

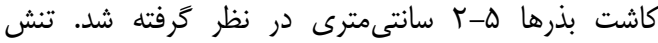

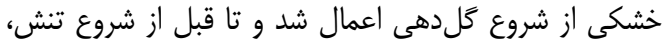

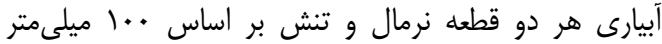

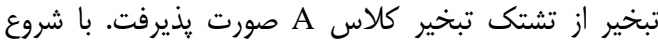

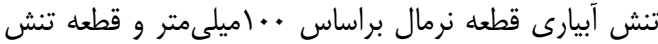
بر اساس •." ميلىمتر تبخير از تشتك كلاس A A انجام كرفت.

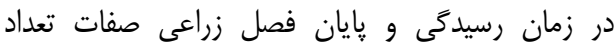

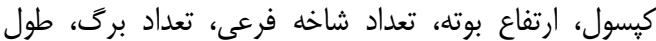

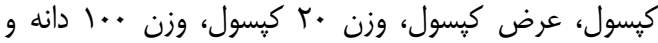

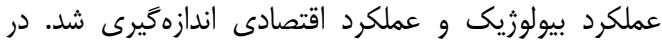

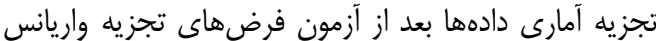

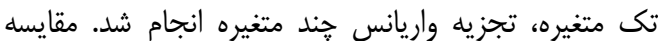

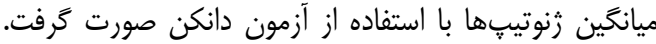

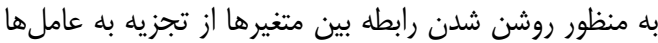

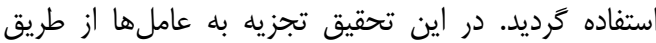

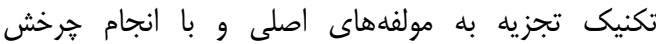

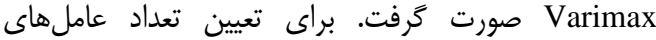

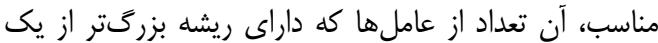

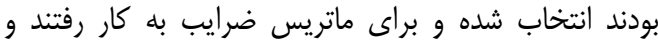

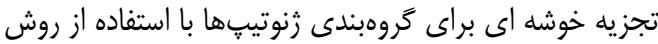

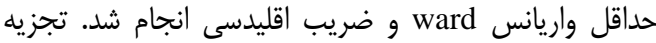

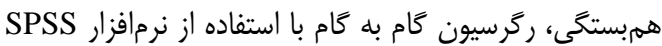

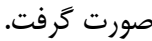

تتايج و بحث تجزي تجزيه واريانس صفات ونت مختلف در شرايط أبيارى بدون تنش و تنش و ور

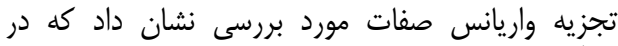

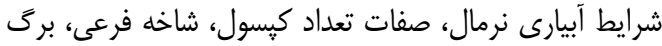

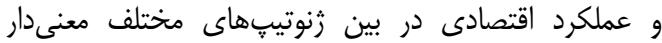

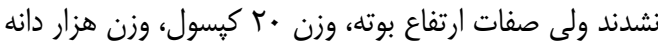

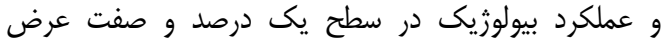

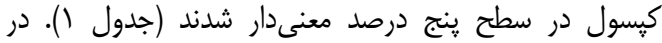

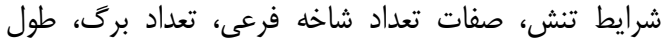

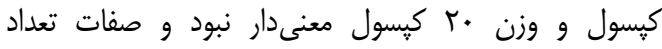

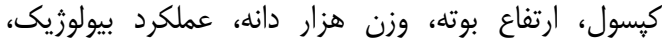

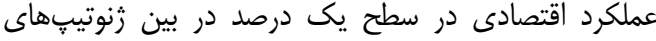

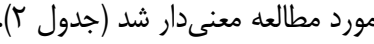

تعداد برگى در بوته، عملكرد بيولوزيكى و عملكرد دانـه كـاهش

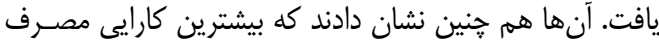

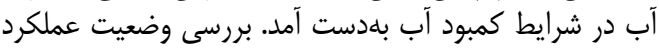

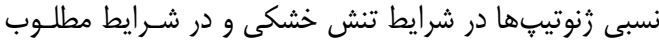

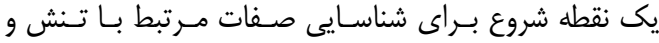

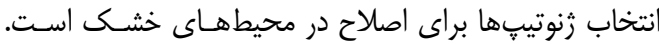

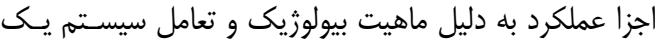

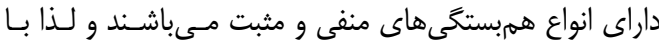

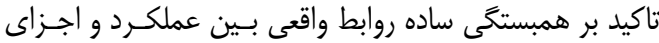

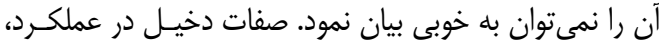

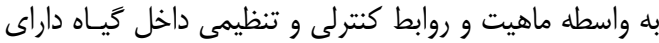

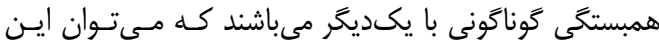

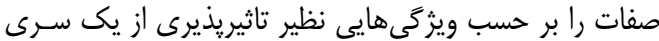

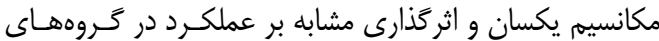
مجزا و غيرهمبسته به نام عوامل (Factor) قرار داد. اين اين شيوه

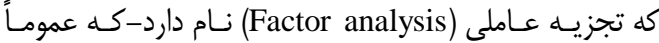

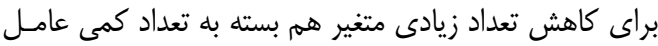

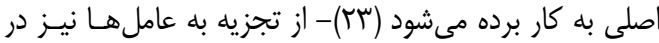

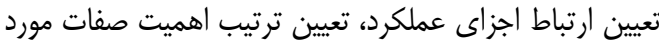

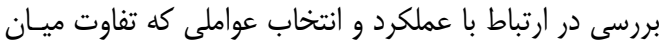

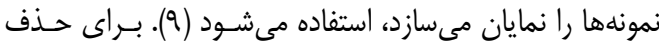

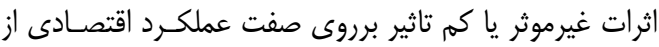

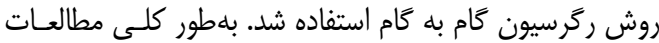

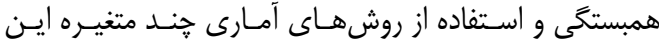

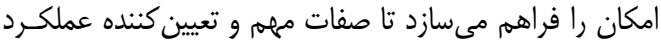

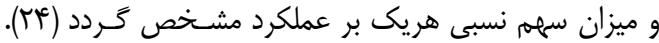

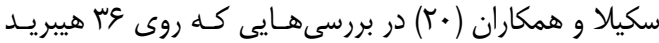

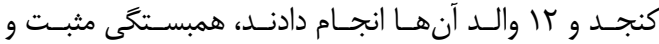

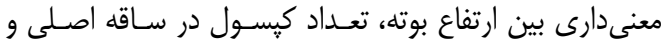

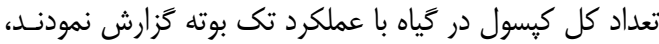

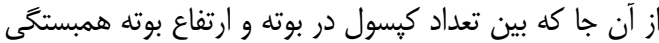

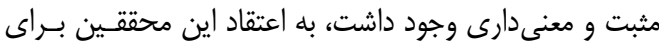

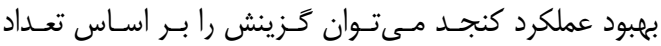

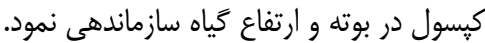

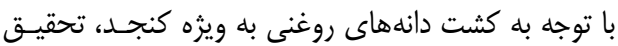

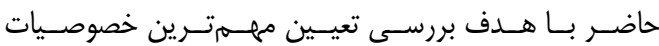

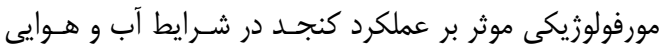

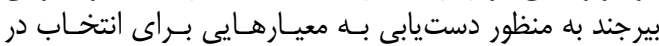

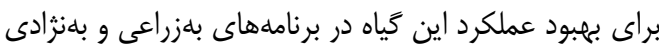
در شرايط منطقه صورت گرفت.

\section{مواد و روشها}

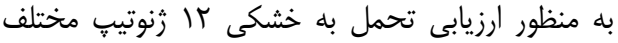

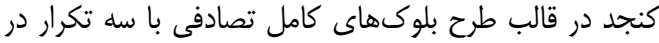

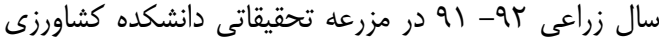

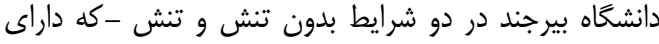



كزينش مهمترين صفات موثر بر عملكرد زنوتيبهاى كنجد

Table 1. Analysis of variance of sesame in normal conditions

جدول ا- تجريه واريانس صفات مختلف كنجد در شرايط نرمال

\begin{tabular}{|c|c|c|c|c|c|c|c|c|c|c|c|}
\hline عملكرد & عملكرد & وزن هزار & وزن •r & عرض & طول & تعداد & تعداد & ارتفاع & تعداد & درجه & منابع \\
\hline اقتصادى & بيولوزيك & دانه & كيسول & كيسول & كسبول & برى & شاخه رعى & بوته & كيسول & آزادى & تغيير \\
\hline$N^{n \text { ns }}$ & सा & $.18^{n+17}$ &.$/ 9^{\prime \prime \prime}$ & $\% \cdot r^{*}$ & $\cdot / \cdot \mathrm{rns}^{\mathrm{ns}}$ & $|\Delta \wedge|^{\mathrm{ns}}$ & $\cdot / \mathrm{v}^{\mathrm{ns}}$ & TYT/S" & $11 \% q / \Delta^{\mathrm{ns}}$ & 11 & رُنوتيب \\
\hline $\mid \Delta N / r^{*}$ & $r N / r^{n s}$ & $\cdot 1 \cdot r^{\mathrm{ns}}$ & $\cdot|\wedge|^{\mathrm{ns}}$ & $\cdot / \cdot 1^{* * *}$ &.$/ 91^{*}$ & $r v r^{\text {ns }}$ & $1 / 0^{\mathrm{ns}}$ & $\mid \varphi / q^{\mathrm{ns}}$ & $\varphi g N / 1^{\text {ns }}$ & $r$ & بلوى \\
\hline$\Delta F / \mathcal{G}$ & 䧃 & $\cdot / r$ & זו/. &.$/ . .1$ &.$/ 19$ & $11 / 99 / r$ & $\cdot / \Delta$ & $\Delta \cdot / \mathcal{F}$ & $r \mu / r$ & r & خطا \\
\hline
\end{tabular}

Table 2. Analysis of variance of sesame in stress conditions

\begin{tabular}{|c|c|c|c|c|c|c|c|c|c|c|c|}
\hline اقتصادى عملكرد & بيولوزيك عملكرد & وزن هزار & كيسول & كيسول & كسيول & تعداد & تعداد شاخه & ارتفاع & كيسول & آزادى درجه & منابع تغيير \\
\hline$r \cdot / V e^{* * *}$ & sr. $/ \cdot M^{* * *}$ & $\cdot / \mathrm{v} \cdot \cdot \mathrm{vN}^{* * *}$ & $\cdot \mid \Delta N v^{\mathrm{ns}}$ &.$/ .11^{\mathrm{ns}}$ & $\cdot / \cdot 118^{\mathrm{ns}}$ & $\Lambda \cdot r / 8$ &.$/ q T^{n s}$ & $T V \cdot / r V^{* * *}$ & $\Delta \cdot / r r^{* * *}$ & 11 & رنوتيڤهاى \\
\hline$\cdot|q q|^{\mathrm{ns}}$ & $r / N \in)^{n s}$ & $\cdot / \cdot \cdot q \cdot r^{\mathrm{ns}}$ & $.1 .4+10^{* * *}$ & $\cdot\left(\cdot .1 \cdot 1^{\mathrm{ns}}\right.$ & $\cdot / \cdot 1 v^{\mathrm{ns}}$ & $\begin{array}{l}11 \\
\text { IDFY }\end{array}$ & $r / \Lambda^{* * *}$ & $\mid F \varphi / \kappa T^{n s}$ & $|r / T|^{* * *}$ & $r$ & بلوى \\
\hline$r / \Lambda Q$ & $r \Delta / \cdot \varphi$ & $.1 . .9$ & .1 .14 &.$/ . .1$ & .1 .1 & $r \Delta Q / 1$ &.$/ 40$ & $v^{e} / 4$. & $1 . / \% 1$ & rr & خطا \\
\hline
\end{tabular}

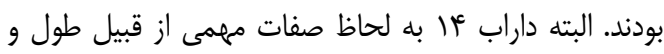

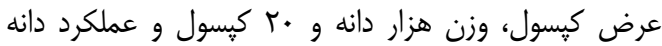

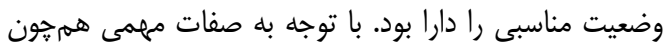

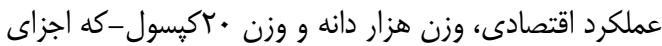

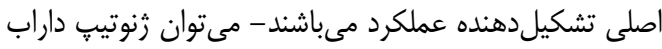

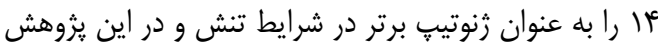

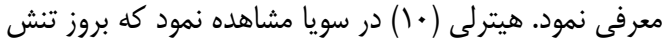

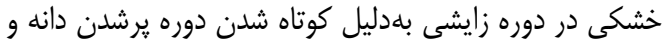

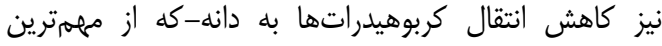

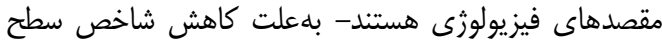

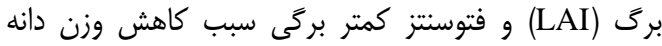

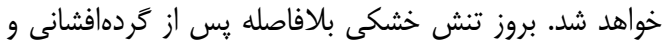
در نتيجه ريزش دانه از عوامل موثر بر بر وزن هزارل برار دانه هستند
مقايسه ميانكَين زنوتيبهاى مختلف كنجد در شرايط

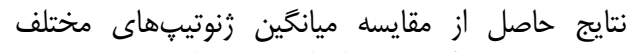

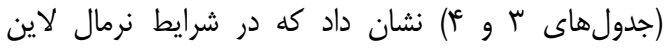
TN234

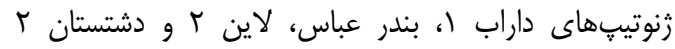

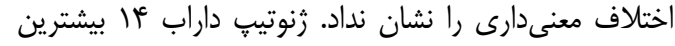

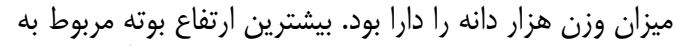

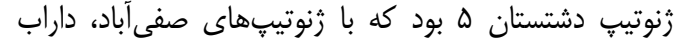

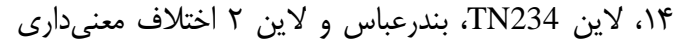

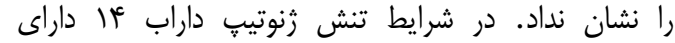

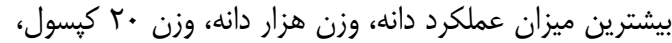

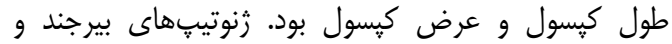

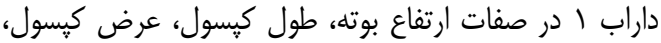

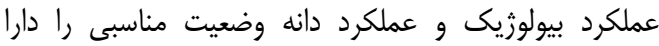

Table 3. Mean comparison of different genotypes of sesame in normal conditions

جدول بـ مقايسه ميانگين ثنوتيبهاى مختلف كنجد در شر ايط نرمال

\begin{tabular}{|c|c|c|c|c|c|c|c|c|c|c|c|c|}
\hline دشتستان r & لاين r & يلووايت & خوسف & بندرعباس & $\begin{array}{l}\text { لاين } \\
\text { TN234 }\end{array}$ & تربت جام & داراب If & صفى آباد & دشتستان ه & داراب ا & بيرجند & رنوتيِهاى \\
\hline$V \Delta / T \varepsilon^{\mathrm{de}}$ & $\mathrm{VN} / \mathrm{r}^{\mathrm{c}-\mathrm{e}}$ & $V^{c} / \Delta r^{\mathrm{de}}$ & $V \Delta / V g^{e d}$ & $\Lambda Y / q Y^{a b}$ & $94 / \Delta \xi^{a b}$ & $9 \Delta / \Delta \cdot \cdot^{e}$ & $\Lambda T / / F^{b-a}$ & $q \cdot / \Delta \gamma^{a-c}$ & $q s / r k^{a}$ & $\Lambda \mathrm{N} / \wedge \mathrm{e}^{\mathrm{cd}}$ & $\Lambda \varepsilon / V^{r^{a-a}-a}$ & ارتفاع بوته \\
\hline$r / 4 \xi^{a b}$ & $r / \Delta \gamma^{\mathrm{ab}}$ & $\Gamma / \Delta *^{a b}$ & $r / v \cdot{ }^{a}$ & $r / \Delta \Lambda^{a b}$ & $r / \& q^{a}$ & $T / T^{D}$ & $r / q r^{\mathrm{a}}$ & $r / F F^{a b}$ & $r / q^{a}$ & $\Gamma / \Delta \mathcal{F}^{\mathrm{ab}}$ & $r / \Delta F^{a b}$ & طول كين \\
\hline - $/\left.\Delta\right|^{\mathrm{a}-\mathrm{c}}$ & $\cdot / \Delta \mathrm{V}^{\mathrm{a} D}$ & $\cdot / \Delta \mathrm{V}^{\mathrm{ab}}$ & $\cdot \mid \Delta f^{a-c}$ & $\cdot / \Delta r^{\mathrm{a}-\mathrm{c}}$ & $\cdot(\Delta)^{\mathrm{C}}$ &.$/ 19^{\circ}$ & $\cdot / \Delta \Lambda^{a}$ & $\cdot / \Delta \mathrm{V}^{\mathrm{aD}}$ & $\cdot \angle \Delta D^{\mathrm{aD}}$ & $\cdot / \Delta \Lambda^{a}$ & $\cdot /\left.\Delta\right|^{\mathrm{c}-\mathrm{c}}$ & \\
\hline$T / V \Delta^{a-c}$ & $T / T \xi^{\mathrm{c}-\mathrm{e}}$ & $\mathrm{r} / \mathrm{1}^{\mathrm{de}}$ & $T / M \Lambda^{c-e}$ & $r / h^{-} \cdot{ }^{D-e}$ & $r / q r^{a-a}$ & $r / 1 \cdot e^{c-e}$ & $r / \cdot 1^{\text {ab }}$ & $1 / 9 .^{e}$ & $r / r v^{a}$ & $t / T T^{c-e}$ & $1 / \Lambda r^{e}$ & \\
\hline$T / T \omega^{\mathrm{ca}}$ & $1 / 18^{e-g}$ & $1 / 99^{g}$ & $r / \cdot r^{a-I}$ & $T / \Delta V^{D}$ & $1 / 9)^{e-g}$ & $1 / 91^{c-e}$ & $\Gamma / \Gamma \omega^{\mathrm{a}}$ & $1 / v 9^{I g}$ & $r / r \varepsilon^{D c}$ & $T / \cdot \mu^{a-I}$ & $1 /\left.\lambda\right|^{\mathrm{Ig}}$ & وزن هزار دانه \\
\hline $\mid r q / \varepsilon g^{a b}$ & a س & $|r| / q V^{a b}$ & ا & 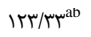 & & $9 / / r r^{c}$ & $119 / \mu^{0}$ & $1 . .^{c}$ & 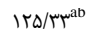 & $1 r \cdot / .^{a b}$ & $119 / 9 q^{a b}$ & عملكرد بيولوزيك \\
\hline$r \in / F^{\mathrm{a}-\mathrm{c}}$ & $\mid F / r r^{c}$ & $19 / 1 e^{\mathrm{a}-\mathrm{c}}$ & $19 / 1 r^{\mathrm{a}-\mathrm{c}}$ & $r V / r \Lambda^{a d}$ & $r \Delta / T \varepsilon^{\mathrm{a}-\mathrm{c}}$ & $\mid V / G \cdot^{\mathrm{a}-\mathrm{c}}$ & $r q / \varphi^{a}$ & $11 / 9 \mu^{\mathrm{c}}$ & $r / / I^{\mathrm{a}-\mathrm{c}}$ & $r F / \wedge q^{\mathrm{a}-\mathrm{c}}$ & $19 / 91^{\mathrm{a}-\mathrm{c}}$ & عملكرد اقتصادى \\
\hline
\end{tabular}
اختلاف ستون هايى كه داراى حروف الفبايى مشابه هستند از لحاظ أمارى و در سطح ه درصد (LSD) معنى دار نيست.

Table 4. Mean comparison of different genotypes of sesame in stress conditions

\begin{tabular}{|c|c|c|c|c|c|c|c|c|c|c|c|c|}
\hline دشتستان r T & لاين r & يلووايت & خوسف & بندرعباس & لاين ع بr & تربت جام & داراب fl & صفى آباد & دشتستان ه & دارابا & بيرجند & رثنوتيصها \\
\hline$g g / r^{0-a}$ & $\Lambda \cdot / \mu^{\mathrm{alo}}$ & $\Delta \mathrm{V} / \mathrm{r}^{\mathrm{Cd}}$ & $9 \Delta / r^{\mathrm{Ca}}$ & $\mathrm{V} / \mathrm{r}^{\mathrm{ado}}$ & $V \cdot / V^{\mathrm{a}-\mathrm{c}}$ & $\Delta f / T^{a}$ & $11 / \wedge^{\mathrm{a} o}$ & $V \Delta / \Delta^{\mathrm{ab}}$ & $\Lambda r / q^{a}$ & $9 \Delta / q^{D-a}$ & $V \Delta / \Delta \cdot{ }^{a D}$ & ارتفاع بوته \\
\hline$\cdot / \Delta \Lambda^{a \mathrm{D}}$ & $\cdot / \Delta \wedge V^{a}$ & . $/ \Delta r^{a b}$ & $\cdot / \Delta \mathrm{r}^{\mathrm{ab}}$ & $\cdot \mid \Delta \& \mathrm{~V}^{\mathrm{a}-\mathrm{c}}$ & $\cdot / \Delta \Lambda^{a b}$ & $\cdot / \Delta \omega^{a b}$ & $\cdot / \Delta \Delta^{\mathrm{ab}}$ & $\cdot / \Delta \Delta^{\mathrm{ab}}$ & $\Delta T . . / .^{D}$ & $\cdot / \Delta V^{a b}$ & $\cdot / \Delta \Delta^{\mathrm{ab}}$ & عرض كيسول \\
\hline$r / \cdot V^{c}$ & $1 / \Delta \Lambda^{\mathrm{ae}}$ & $r / \cdot r^{c}$ & $r / \cdot \Delta^{c}$ & $T / 4 I^{D}$ & $r / .^{c}$ & $1 / 99^{c}$ & $r / \varphi^{\mathrm{a}}$ & $1 / q^{c} \gamma^{c}$ & $T / F D^{D}$ & $1 / \Delta q^{\mathrm{de}}$ & $1 / r^{a}$ & \\
\hline $1 / \wedge \Delta^{c}$ & $1 / 4 \varepsilon^{I}$ & $1 / r^{g}$ & 1/צrae & $T / K I^{D}$ & $1 / \Delta 1^{\mathrm{eI}}$ & $1 / v^{c a}$ & $r / Q \Lambda^{\mathrm{a}}$ & $1 / q^{g}$ & $T / 19^{\circ}$ & $1 / 9 e^{\mathrm{de}}$ & $|/ \Delta|^{\mathrm{er}}$ & وزن هزار دانه \\
\hline $\mid r V / g^{\mathrm{ab}}$ & $\mid r N / g^{a b}$ & $119 / 8^{\circ}$ & ११/४ ${ }^{a}$ & $1 r \cdot / q^{\mathrm{ab}}$ & $r q / \mu^{a}$ & $91 / *^{c}$ & $11 \cdot / 9^{\mathrm{c}}$ & $19 / 9^{c}$ & $11 \cdot / \mu^{c}$ & $\mid \mathrm{rN} / \cdot^{\mathrm{ab}}$ & $11 \cdot / \mu^{c}$ & عملكرد بيولوزيك \\
\hline $10 / \&^{c a}$ & $\mid r / \cdot r^{\text {ae }}$ & $|V / f|^{D C}$ & סc & $\mid r / \Delta r^{\mathrm{de}}$ & $|N / \Delta|^{\text {ab }}$ & $\mid w / / v^{\text {de }}$ & & a & $1 f / 9 .{ }^{c-e}$ & $\mid r / A^{A C}$ & $\mid r / r \cdot{ }^{e}$ & عملكرد اقتصادى \\
\hline
\end{tabular}




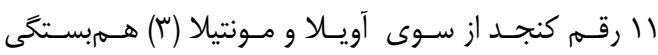

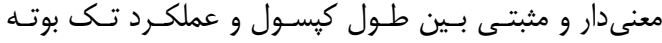

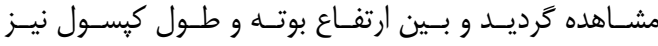

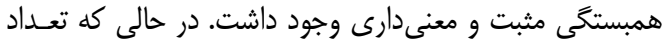

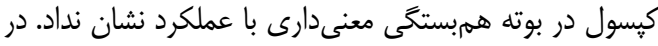

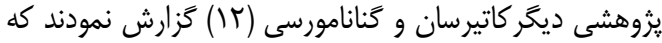

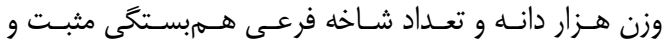

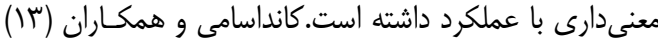

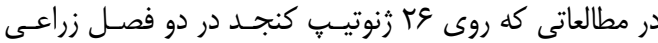

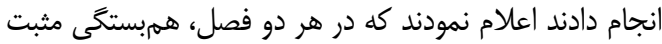

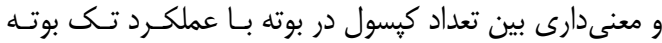

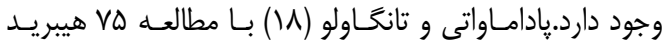

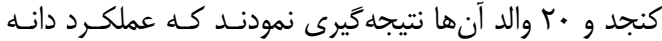

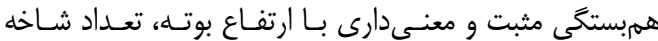

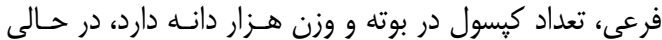

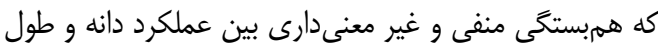

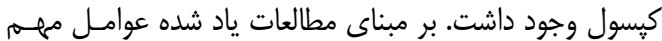

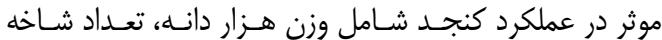

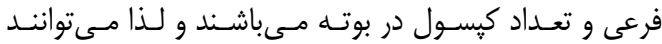

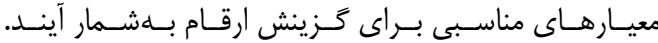

تجزيه همربستكى صفات مختلف در شرايط بدون تنش

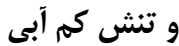

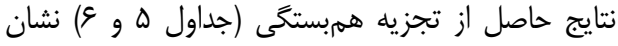

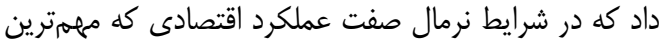

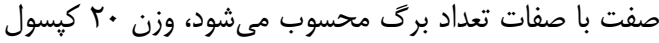

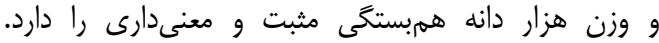

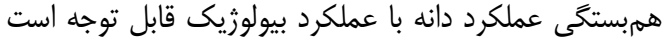

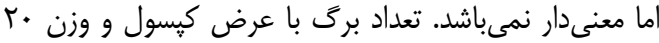

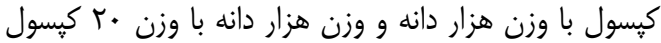

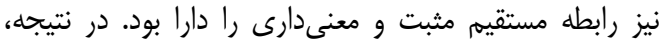

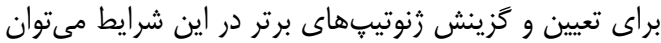

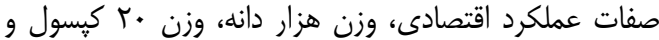

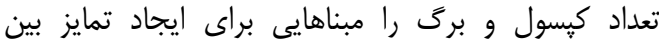

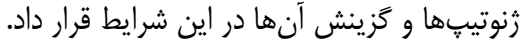

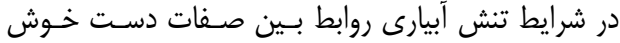

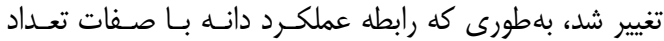

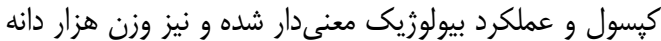

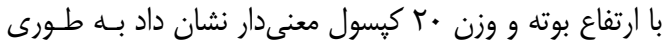

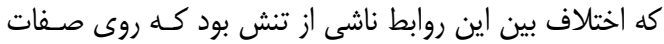

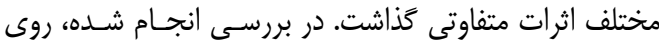

جدول ه- سطوح معنى دارى همبستخى صفات مختلف زنوتيڤ هاى كنجددر شرايط نرمال Table 5. Correlation analysis of sesame genotypes in normal conditions

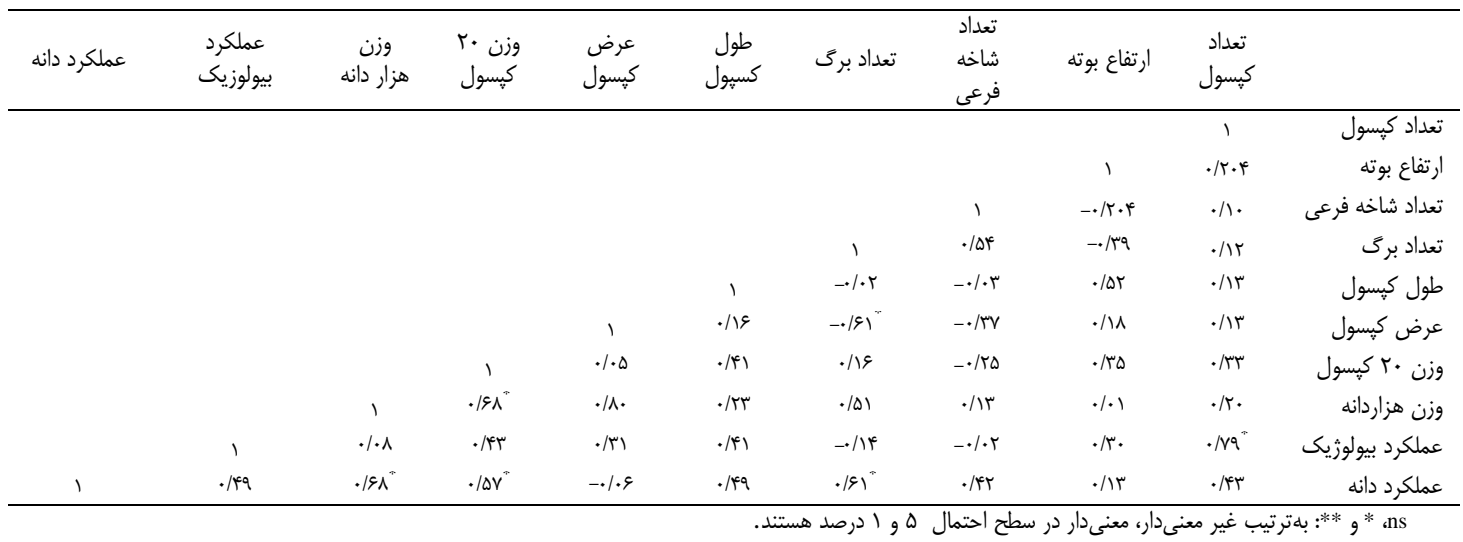

جدول צ- سطوح معنى دارى همبستخى صفات مختلف زنوتيڤهاى كنجد در شرايط تنش

Table 6. Correlation analysis of sesame genotypes in stress conditions

\begin{tabular}{|c|c|c|c|c|c|c|c|c|c|c|}
\hline عملكرد دانه & عملكرد بيولوزيك & وزن هزار & كيسول نr & عرض كيسول & كسيول & تعداد برى & تعداد شاخه & بوتف & تعداد كֶسول & \\
\hline & & & & & & & & & 1 & تعداد كֶيول \\
\hline & & & & & & & 1 &.$/ \cdot v$ &.$/ T \Lambda$ & تعداد شاخه فرعى \\
\hline & & & & & & 1 & $\cdot / \Delta \cdot{ }^{* * *}$ & $\cdot / r$. & r & تعداد برگ \\
\hline & & & 1 & 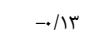 & - n & $\cdot / T F$ & -.1 .9 &.$/ 19$ & $\rightarrow / T r$ & وزن •r كيسول \\
\hline & & 1 & $\cdot / \Lambda \varepsilon^{*}$ & $-* / 1$ & $-.1 \cdot \Delta$ & $\cdot / 19$ & - &.$/ \% q^{*}$ & $-\cdot / \mu+-$ & وزن هزاردانه \\
\hline & 1 & .1 .1 & .1 .1 & $\cdot / \pi V$ & $\cdot / \pi F$ &.$/ . \Delta$ & $.1 \cdot 1$ & .1 .9 & .110 & عملكرد بيولوزيك \\
\hline 1 &.$/ \% q^{*}$ & ع &.$- / 18$ &.$/ 14$ &.-+1 &.$/ 10$ &.$/ 19$ &.$- / 4 r$ & $\cdot / \Delta f^{* \pi *}$ & عملكرد دانه \\
\hline
\end{tabular}


ضريب معنى دار ركرسيونى رادر تبيين عملكرد از خود نشان

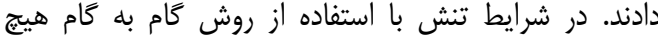

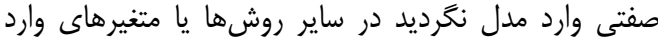

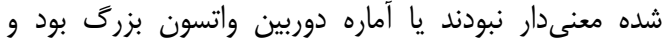

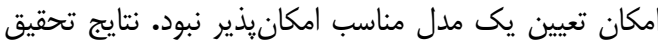

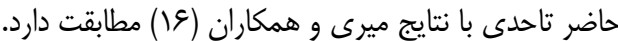

تجزيه رتر سيون صفات در شرايط نرمال و تنث بن

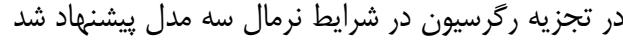

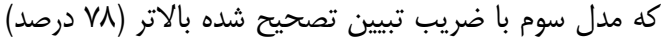

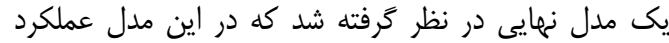

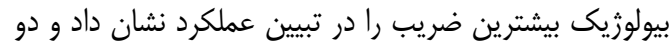

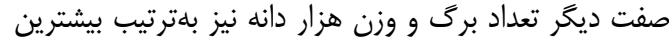

جدول Y- تجزيه ركر سيون صفات مختلف زنوتيبهاى كنجد در شرايط نرمال Table 7. Regressionanalysis of sesame genotypes in normal conditions

\begin{tabular}{|c|c|c|c|c|c|}
\hline معنى دارى & t أماره & ضريب ركر سيون & خطاى معيار & ضرايب ركر سيون استاندارد نشده & جزاى مدل \\
\hline.$/ . .9$ & $-r / F r$ & - & $9 / \Gamma r$ & $-r \mid / \Lambda$ & تابت \\
\hline 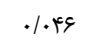 & $r / \pi \Delta$ & qq q/. & 1/9ه & $F / 9$. & وزن هزار دانه \\
\hline $.1 . .9$ & $r / v$. &.$/ \Delta r$ & .1 .9 &.$/ \pi r$ & عملكرد بيولوزيك \\
\hline.$/ .11$ & r/qF &.$/ 49$ &..$r$ &.$/ 1$ & تعداد بركى \\
\hline
\end{tabular}

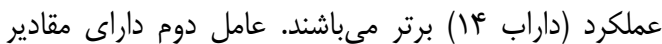

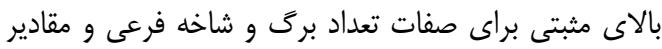

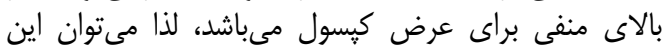

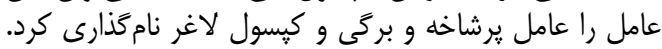

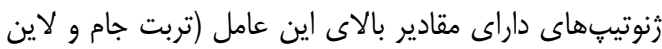

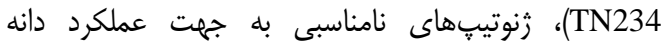

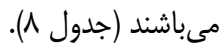

تجزيه به عاملهاى صفات مختلف در شرايط نرمال

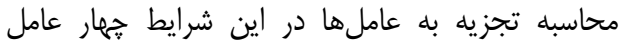

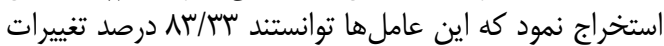

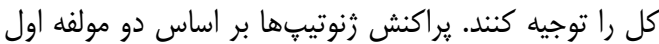

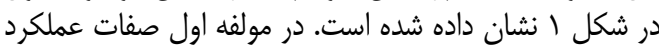

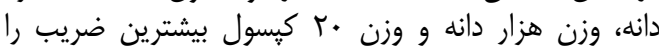

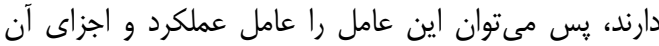
نام كذارى كرد و زنوتيبهاى باى با مقادير بالاى اين عاين عامل داراى آن

جدول ^- تجزيه به مولفههاى اصلى صفات مختلف زنوتيڤهاى كنجد در شرايط نرمال Table 8 . The main component analysis of different traits sesame genotypes in normal conditions

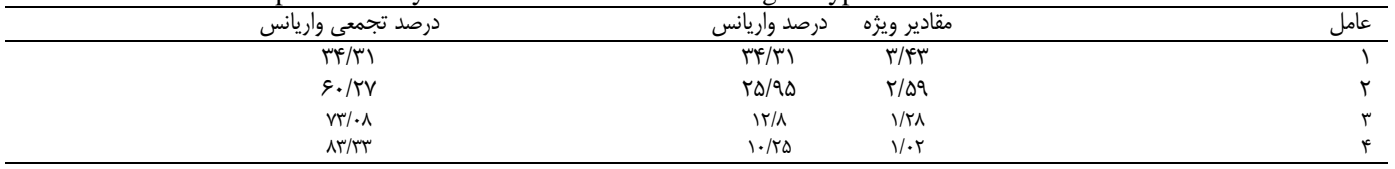

جدول 9- تجزيه به عاملهاى صفات مختلف زنوتيبهاى كنجد در شرايط نرمال Table 9. Factor analysis of the different traits of sesame genotypes in normal conditions

\begin{tabular}{|c|c|c|c|c|}
\hline عامل |F & عامل ب & عامل & عامل & \\
\hline.$/ .1$ &.$/ 91$ &.$/ \%$ &.$/ 1 \Delta$ & تعداد كيسول \\
\hline$\cdot / \Lambda \Delta$ &.$/ 1$ & $\cdot(r)$ &. .1 .1 & ارتفاع كَياه \\
\hline.$- / \cdot v$ &.$/ M$ & $\cdot / A F$ & -.1 .9 & تعداد شاخه فرعى \\
\hline$-\pi / \pi$ & $\rightarrow / .4$ &.$/ W r$ &.$/ \Delta$. & تعداد برى \\
\hline /Ar & זו/. & .1 .4 &.$/ \pi \Delta$ & طول كيسول \\
\hline .1 .4 &.$/ r q$ & $-/ / v \Delta$ &. .4 & عرض كيسول \\
\hline 低 &.$/ 19$ &.$- / 14$ & $\cdot|\Lambda|$ & وزن هزار دانه \\
\hline. .1 .1 &.$/ .4$ &.$/ 1$ &.$/ 99$ & وزن •r كيسول \\
\hline.$/ r 9$ &.$/ 9$. & - &.$/ 1$ & عملكرد بيولوريك \\
\hline . & Tא/. & 的 &.$/ 91$ & عملكرد اقتصادى \\
\hline
\end{tabular}




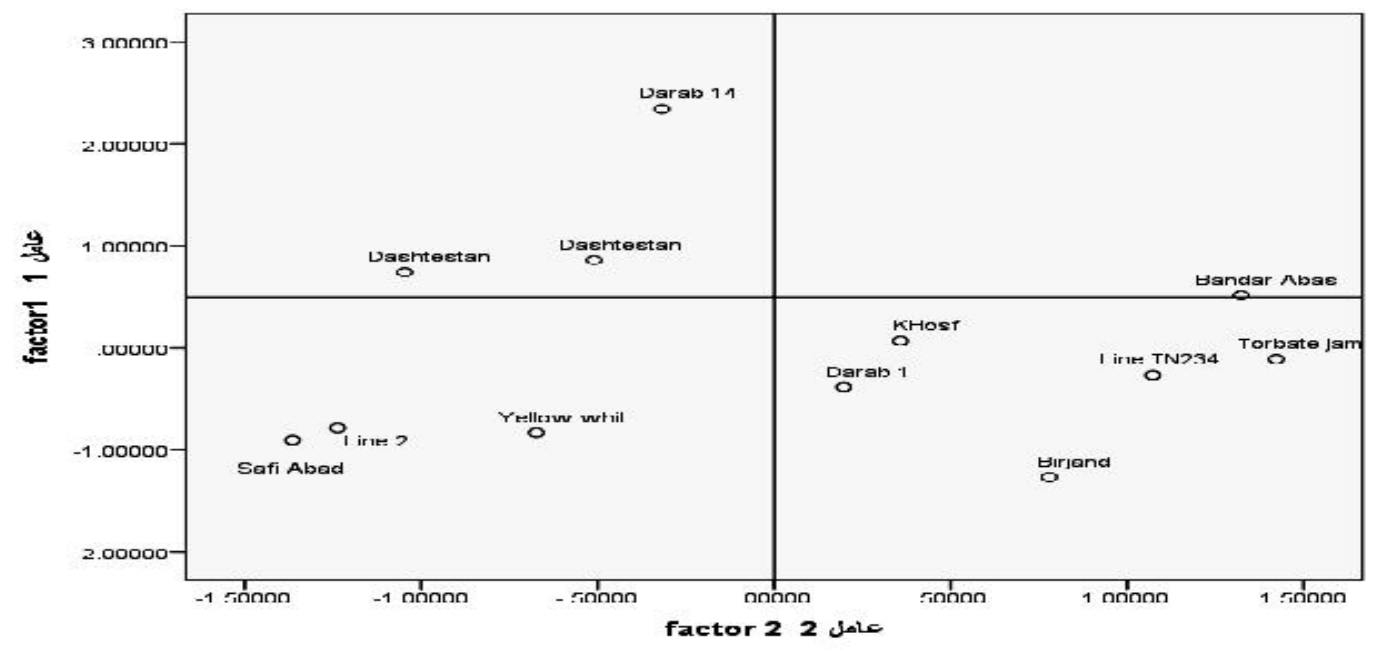

شكل ا- يراكنش زنوتيٍهاى مختلف كنجد بر اساس عاملهاى حاصل از تجزيه به عاملها در شرايط نرمال

Figure 1. The distribution of different genotypes of sesame based on the operating results of the factor analysis in normal conditions

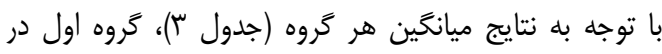

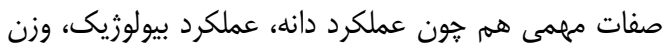

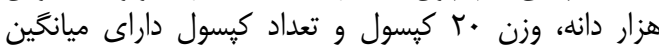

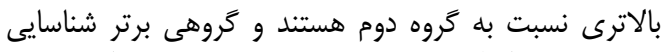

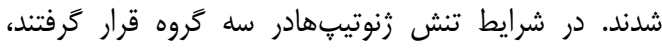

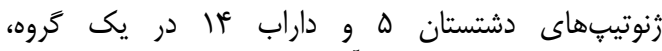

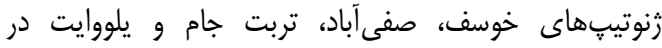

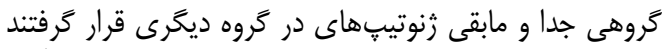

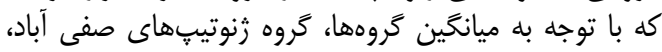

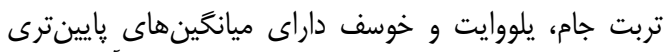

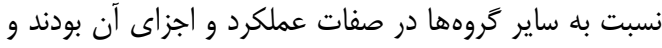

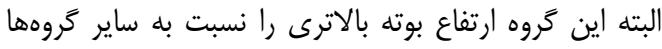

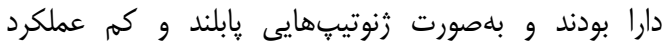

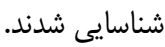

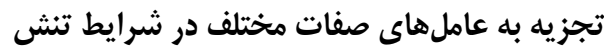

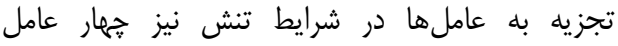

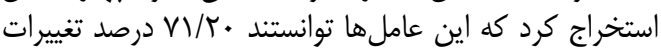

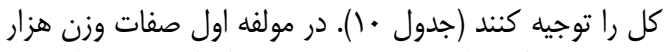

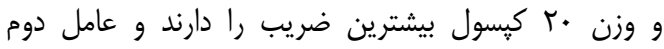

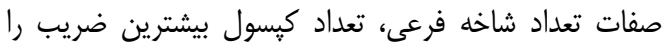

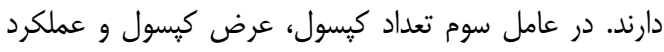

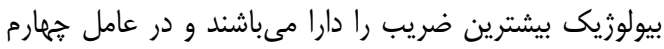
صفات ارتفاع بوته، طول كيسول و و عرض كيسول ديسول بيشترين

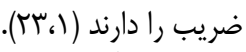
تجزيه خوشهاى صفات مختلف در شرايط نرمال وال و تنش

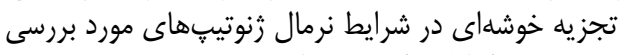

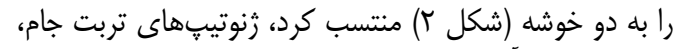

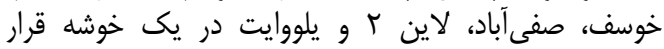

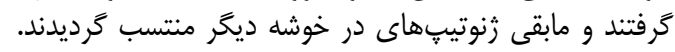

جدول • (- تجزيه به مولفههاى اصلى صفات مختلف زنوتيٍٍاى كنجد در شرايط تنش Table 10. The main component analysis of different traits sesame genotypes in stress conditions

\begin{tabular}{|c|c|c|c|}
\hline درصد تجمعى واريانس & درصد واريانس & مقادير ويزه & عامل \\
\hline$r . / 91$ & $r . / 91$ & $r / \& q$ & 1 \\
\hline$r .1 . r$ & $19 / 1 \%$ & $1 / 90$ & $r$ \\
\hline$\Delta N / \Gamma$ & W/TA & $1 / q \mu$ & r \\
\hline$V / r$. & $1 r / 19$ & $1 / \mu$ & f \\
\hline
\end{tabular}


جدول Iا- تجزيه به عاملهاى صفات مختلف زنوتيبهاى كنجد در شرايط تنش Table 11. Factor analysis of the different traits of sesame genotypes in normal conditions

\begin{tabular}{|c|c|c|c|c|}
\hline عامل Fe & عامل r & عامل r & عامل 1 & \\
\hline.$- / 199$ & . & . /FTt & -•/rqq & تعداد كيسول \\
\hline . /Ar. & $\cdot 11 \cdot 4$ & . MFV & $\cdot / M M$ & ارتفاع كَياه \\
\hline $.1 .4 \mathrm{~T}$ &.$- / . r V$ & . $/ \wedge r q$ &.$- / 1 T \Delta$ & تعداد شاخه فرعى \\
\hline.$/ 1 \cdot 1$ &.$- / . \mu l$ & $\cdot / \mathrm{VrV}$ & . $/ T H V$ & تعداد برى \\
\hline$\cdot / V^{\mu}$ &. $\mid g 4 y^{4}$ & $-. / 4+1$ &.$- / 148$ & طول كيسول \\
\hline $.1 .9 \mathrm{~V}$ & 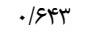 &.$- / Q T$ &.$- / 11$. & عرض كيسول \\
\hline . & - &.$/ 9$. &.$/ 940$ & وزن هزار دانه \\
\hline$\cdot / \Gamma / Q$ &.$- / .4 T$ & $\cdot / / V$ & . /QTT & وزن •r كيسول \\
\hline.$- / / \mathrm{V}$. & $\cdot / V<q$ &.$/ 1 T V$ & ./ALT & عملكرد بيولوريك \\
\hline.$- / 1099$ &.$/ 491$ & • &.$- / 199$ & عملكرد اقتصادى \\
\hline
\end{tabular}

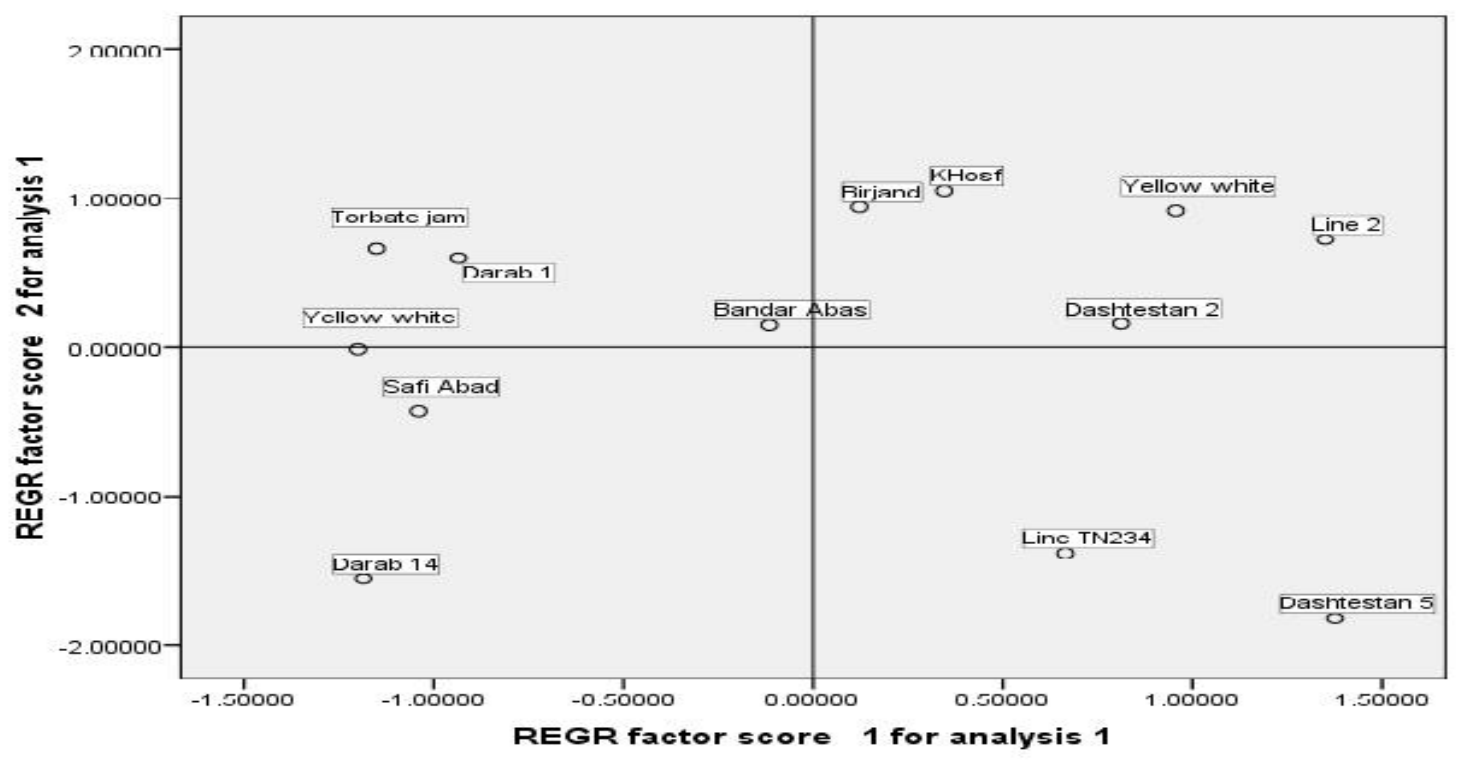

شكل r- يراكنش زنوتيبهاى مختلف كنجد بر اساس عاملهاى حاصل از تجزيه به عاملها در شرايط تنش

Figure 2. The distribution of different genotypes of sesame based on the operating results of the factor analysis in stress conditions

دو صفات با اختلاف معنىدار با ساير زنوتيڤها دارا بود و

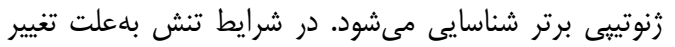

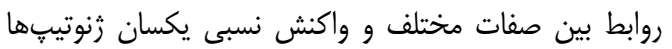

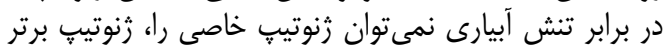

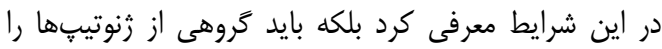
زنوتيڤ برتر در نظر گرفت (شكل ساو
بر اساس نتايج اين تحقيق مىتوان نتيجهَيرى نمود كه

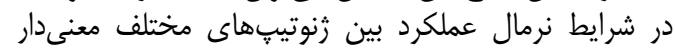

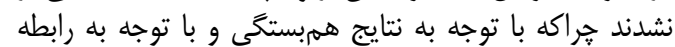

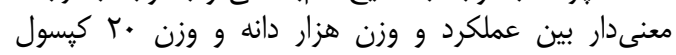

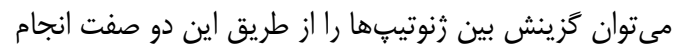

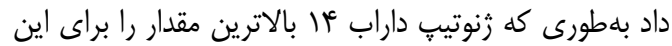




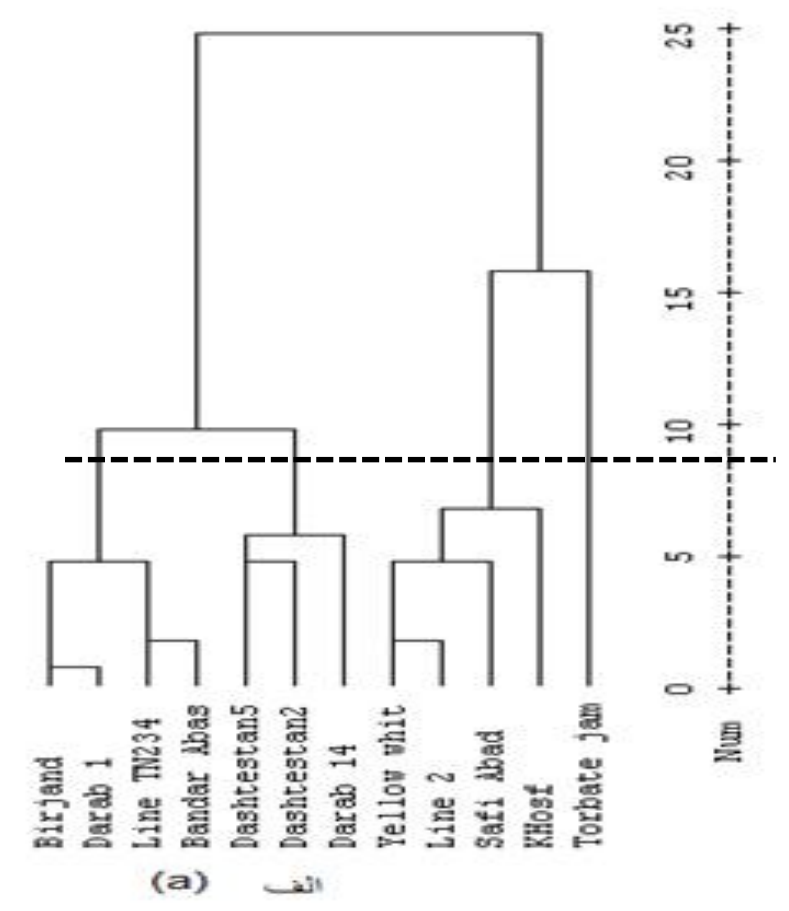

شكل س- تجزيه خوشهاى زنوتيبهاى مختلف كنجد در شرايط نرمال (الف)

Figure 3. Cluster analysis ofdifferent genotypes of sesame in normal conditions

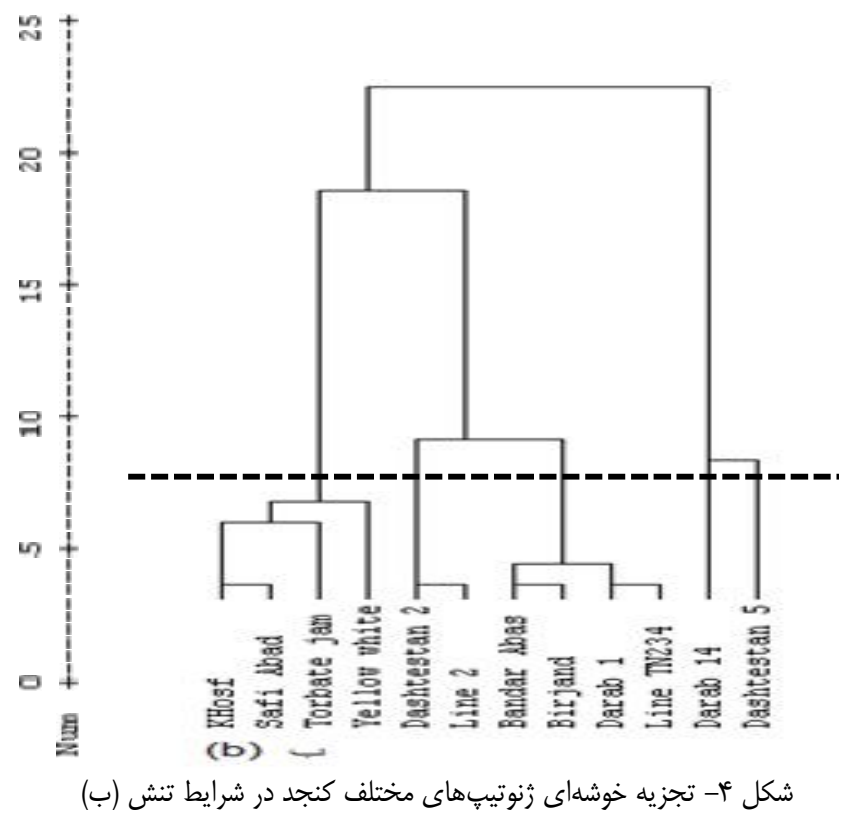

Figure 4. Cluster analysis of different genotypes of sesame in stress conditions 
1. Amini, A., M.R. Qanadha and S. Ebdmishani. 2000. Factor analysis for morphologic and phonological traitsin beans. Seed and Plant, 16: 210-218 (In Persian).

2. Annan, K. 2001. Water for sustainable agriculture in developing regions-more crop for every scarce crop, In: proceeding of $8^{\text {th }}$ JIRCAS International Symposium, 27-28 Nov. 2001. Epochal Tsukuba, Tsukuba Science City, Japan, pp: 132-133.

3. Avila, J. and D. Montilla. 1997. Yield, yield components and tolerance to whitefly (Bemisiatabaci) incidence as sesame (Sesamum indicum). Selection criteria Sesame and Sofflower Newsletter, 12:1421.

4. Blum, A. 1988. Plant Breeding for Stress Environments CRC press, Boca Raton, FL. pp: 38-78.

5. Dilip, K., M. Ajumdar and S. Roy. 1991. Response of summer sesame (Sesamum indicum) to irrigation, row spacing and plant population. Journal Indian Agronomy, 37: 758-762.

6. Eskandari, H., S. ZehtabSalmasi and K. Ghasemi-Golozani. 2010. Evaluation of water use efficiency and grain yield of sesame cultivars as a second crop under different irrigation regimes. Journal of Sustainable Agriculture Science, 2/20: 39-51 (In Persian).

7. FAO. 2011. Bulletin of Statistics, 4: 43-45.

8. Fischer, R.A. and R. Maurer. 1978. Drought resistance in spring wheat cultivar, I: Grain yield responses. Journal Agriculture Research, 29: 897-912.

9. Grafius, J.E. 1978. Multiple characters and correlated response .Crop Science, 18: 931-934.

10. Heathery. N. 1993.Simulation of Soybean seed yield response to irrigation timing: validation of the EPIC model. Journal of Production Agriculture, 230-232.

11. Jain, S., R. Yue-Lioang, L.E. Mei-wang, Y. Ting-Xian, Y. Xiao-Wen and Z. Hong-Ving. 2010. Effect of drought stress on sesame growth and yield characteristics and comprehensive evaluation of drought tolerance. Chinese Journal of Oil Crops Sciences, 4: 42-48.

12. Kathiresan, G. and P. Gnanamurthy. 2000. Studies on seed yield-contributing characters in sesame. Sesame and Sofflower Newsletter, 15: 29-32.

13. Kandasamy, G., V. Manchoram and S. Thangovelu. 1990. Variability of metric traits and character association in sesame in two sesame and Safflower Newsletter, 5: 10-15.

14. Kumar, A., S.T.N. Prasad and U.K. Prasad. 1996. Effect of irrigation and nitrogen on growth, yield/oil content, nitrogen uptake and water-use of summer sesame (Sesamum indicum) Journal, Indian Agronomy, 41: 111-115.

15. Mensah, J.K., B.O. Obadoni, P.G. Eroutor and F. Onome-Irieguna. 2006. Simulated flooding and drought effects on germination, growth and yield parameters of sesame (Sesame indicum L.), Journal Biotechnology, 5: 1249-1253.

16. Miri, H.R. and Q. Nurmohamdi. 2007. Evaluation of physiological traits on grain yield in Brassica in Fars Province Agricultural Science, 17: 117-101 (In Persian).

17. Quarrie, S.A., J. Stojanovic and S. Pekic. 1999. Improving drought tolerance in small-grain cereals: A case study, progress and prospect, Plant Growth Regulation, 29: 1-21.

18. Padmavathi, N. and S. Thangavelu. 1996. Association of various yield components in sesame. Sesame and Sofflower Newsletter, 11: 40-45.

19. RezvaniMoghaddam, P., Gh. Norozpoor, J. Nabati and A.A. Mohammad Abadi. 2005. Effect of different irrigation intervals and plant density on morphological characteristics, grain and oil yields of sesame (Sesameum indicum L.). Iranian Journal of Agricultural Sciences, 3: 57-68 (In Persian).

20. Sakila, M.S., M. Ibrahim, A. Kalamani and M. Backiyarani. 2000. Correlation studies in sesame (sesamum indicum L.). Sesame and Sofflower Newsletter, 15: 26-28.

21. Salehpour, M., A. Ebadi, M. Izadi and S. Jamaati-e-Somarin. 2009. Evaluation of water stress and nitrogen fertilizer effects on relative water content, membrane stability index, chlorophyll and some other traits of lentils (Lens culinaris L.) under hydroponics conditions. Journal Environment Science 3: 103-109 (In Persian).

22. Shokohfar, A. and S. Yaghubinezhad. 2012. The effect of drought stress on yield components of Sesame (Sesumum indicum L.) cultivars. Agronomy and Plant Breeding Journal, 8: 19-29.

23. Walton, P.D. 1972. Factor and lysis of yield in spring wheat (Triticum aestivum L.) Crop Science, 12: 731-733.

24. Zeynali khanghah, H. and A.R. Sohani. 2008. Effect of Genetic analysis of some agronomic traits in soybean yield by multivariate statistical methods. Journal of agricultural Sciences, Volume3, Number4, pp: 812-807 (In Persian). 


\title{
Choose the Most Important Traits Affecting on Yield of SOME SESAME Genotypes (Sesamum indicum L.) in Normal and Stress Conditions
}

\author{
Afson Askari ${ }^{1}$, Mohammad Zabet ${ }^{2}$, Mohammad Ghader Ghaderi ${ }^{3}$, Ali Reza Samadzadeh ${ }^{3}$ \\ and Ali Shorvazdi ${ }^{4}$ \\ 1-M.Sc. Student, University of Birjand (Corresponding author: askari_2505@yahoo.com) \\ 2 and 3- Assistant Professor and Instructor, University of Birjand \\ 4- Graduate M.Sc., University of Tabriz \\ Received: April 20. $2014 \quad$ Accepted: September 15. 2014
}

\begin{abstract}
Due to evaluation Yield and Its components and determining the most important treats affecting on the Yield, 12 different accessions of sesame were cultivated under both normal irrigation and drought stress by randomized complete block design with three replications at Research Farm of Birjand in 2012-2013 years. The parameters measured included the number of capsules, plant height, number of branches, number of leaves, length and width of capsules, 20 capsules weight, grain weight, the weight of 1000 seeds, biological yield and economic yield. Plant height, 1000 seed weight and biological yield showed significant differences in both irrigated conditions. Darab14regard to important agronomic traitsin both conditions had a suitable situation. The correlation results showed that under normal irrigation, the number of leaves per plant $\left(0.616^{* *}\right), 1000$ seed weight $\left(0.688^{*}\right)$ and weight of 20 capsules $\left(0.579^{*}\right)$ had the highest correlation with grain yield. The regression result showed that Biological yield, Number of leaves and weight of 1000 seed had highest coefficients at justifying the yield, respectively. Factor analysis revealed that four factors more than 83 and $71 \%$ of total variations in all data are justified in normal and stress conditions. A cluster analysis of accessions, divided them into two and three groups in normal and stress irrigation respectively.
\end{abstract}

Keywords: Cluster analysis, Correlation analysis, Factor analysis, Regression analysis, Sesame 